\title{
Application of ALO-ELM in Load Forecasting Based on Big Data
}

\author{
Ming He ${ }^{1,}$, , $\mathrm{Yi} \mathrm{Li}^{1}$, Wan Zou ${ }^{1}$, Xiangxi Duan ${ }^{1}$ \\ 1 State Grid Sichuan Electric Power Co., Ltd., Chengdu 610041, China \\ * Correspondence: 2708340076 @qq.com; Tel.: +86-19923139980
}

\begin{abstract}
The load of power system changes with the development of economy, short-term load forecasting play a very important role in dispatching and management of power system. In this paper, the Ant Lion Optimizer (ALO) is introduced to improve the input weights and hidden-layer Matrix of extreme learning machine (ELM), after the parameters of ELM are optimized by ALO, then input nodes, hidden layer nodes and output nodes are determined, so a load forecasting model based on ALO-ELM combined algorithm is established. The proposed method is illustrated based on the historical load data of a city in China. The results show that the average absolute error of short-term load demand predicted by ALO-ELM model is 1.41, while that predicted by ELM is 4.34, the proposed ALO-ELM algorithm is superior to the ELM and meet the requirements of engineering accuracy, which proves the effectiveness of proposed method.
\end{abstract}

Keywords: load forecasting; extreme learning machine (ELM); ant lion optimizer (ALO); parameter optimization; model.

\section{Introduction}

With the development of technology and civilization, human beings are eager to grasp the trend of development and change. Prediction has become the main research field of machine learning. Power load forecasting is to establish the relationship between future load demand and historical load data, impact factors at a specific time [1].Accurate load forecasting can provide reference for operation, power generation, power distribution, power transmission and system planning and dispatching, power price balance and so on [2]. At the same time, the accurate load forecasting can provide references for the electricity price adjustment of the power supply enterprise, and promote the reform and development of the electricity market [3].

After many scholars and research unceasing exploration, many valuable research results have been obtained in load forecasting aspect. A short-term load forecast method was proposed based on artificial neural network and artificial bee colony algorithms in [4]. A combined method for the short-term load forecasting of electric power systems was presented in [5], which was based on the Fuzzy c-means (FCM) clustering, particle swarm optimization (PSO) and support vector regression (SVR) techniques . A method was proposed to utilize the universal approximation properties of neural and wavelet networks to determine the function relationship between input variables and outputs [6]. A method was propose for short term load forecasting trained by PSO and Core Vector Regression (CVR)[7]. Based on the historical data, the whale algorithm was applied to optimize the parameters of KELM, and the short-term load-forecasting model was established in [8]. Based on fuzzy data processing, bagging algorithm was introduced to sample new samples and trained fuzzy GBDT to establish load-forecasting model[9].To resolve the problem of short-term power load forecasting, a self-adapting particle swarm optimization (PSO) algorithm was proposed to optimize the error backpropagation (BP) neural network model[10]. The methods mentioned above have their own characteristics, for example, support vector machine is a small sample learning method, it can deal with nonlinear 
problems, and the artificial neural network algorithm has the advantages of strong learning and adaptive ability.

Huang G. B. proposed Extreme Learning Machine (ELM) algorithm in [11]. ELM algorithm has been employed on prediction and large sample learning by randomly giving the hidden layer parameters. However, because the implicit parameters of ELM are generated randomly, a series of non-optimal parameters are generated, which weaken the generalization performance of ELM [12]. At present, the parameter optimization algorithms for ELM include Genetic Algorithm and particle swarm optimization [13]. A hillclimbing method was adopted to optimize the weights and thresholds of extreme learning machines, so as to obtain an optimization model with strong stability and high prediction accuracy, the improved model has faster training speed and more accurate prediction, which is applicable for short-term power load forecasting with a large number of influencing factors and huge data volume[14]. The artificial swarm optimization algorithm was used to select the ELM parameters by Cao J. [15].Particle swarm optimization (PSO) was applied to simulate the behavior of birds, and the optimal solution was obtained by particle iteration. Mutation operator was selected to enhance the diversity of population, and the parallel Algorithm (PIPSO-ELM) was adopt to deal with the big data of power load [16]. Most optimization algorithms obtained by simulating the biological behavior. Mirjalili S. proposed ALO Algorithm in 2015, the results of mathematical experiments show that ALO has better optimization performance [17].

Based on the analysis of the literature, this paper presents a short-term load forecasting method based on extreme learning machine and ant lion Algorithm. ALO was used to optimize the parameters of ELM, the electricity load in Chongqing city were selected to verify the effectiveness and feasibility of the proposed prediction algorithm based on ALO-ELM combined method.

\section{Prediction Algorithm Based on ALO-ELM}

\subsection{The Ant Lion Optimizer-ALO}

The Ant Lion Optimizer-ALO simulates the predation behavior of ant and ant-lion to obtain the best solution. The main body of the algorithm is ants and ant lions. Firstly, the position and fitness value of the ant are initialized. The factors that affect the ant's migration are the boundary limit and the ant-lion trap. When the ant finds a better position, than the ant-lion immediately preys on the ant and takes the place of the ant, after the iteration, the optimal solution of the ant-lion is the elite ant-lion, finally the global optimal solution is obtained [18-19]. The formula is as follows.

Ant Migration formula is

$$
\mathrm{X}(t)=\left[0, \operatorname{cs}\left(2 r\left(t_{1}-1\right), \operatorname{cs}\left(2 r\left(t_{2}-1\right), \ldots, \operatorname{cs}\left(2 r\left(t_{n}-1\right)\right)\right]\right.\right.
$$

In the above equation: $c s$ is the cumulative sum, $n$ is the number of Iterations, and $r(\mathrm{t})$ is

$$
r(t)= \begin{cases}1 & \text { rand }>0.5 \\ 0 & \text { rand } \leq 0.5\end{cases}
$$

Where $t$ is a random number, and rand is a random number with a range of $[0,1]$.

In a bounded space, the location of the ant's $t$-th iteration is

$$
X_{i}^{t}=\frac{\left(X_{i}^{t}-a_{i}\right) \times\left(d_{i}-c_{i}^{t}\right)}{\left(d_{i}^{t}-a_{i}\right)}
$$

When the $i$ variable walk randomly, $d_{i}$ is the maximum, $a_{i}$ is the minimum, $d_{i}^{t}$ is the maximum value of $t$-th iteration, $c_{i}^{t}$ is the minimum value of the $t$-th iteration.

The ant moves around the super ball around the ant lion, and affected by the ant-lion trap. The motion formula is

$$
c_{i}^{t}=\text { Antlion }_{j}^{t}+c^{t}
$$




$$
d_{i}^{t}=\text { Antlion }_{j}^{t}+d^{t}
$$

Where $c^{t}, d^{t}$ is the minimum and maximum value of the $t$-th iteration, Antlion $_{j}^{i}$ is the position of the ant-lion $j$ in $t$-th iteration.

As the travel radius of ant decreases, $C$ and $D$ decrease as the number of iterations increases [18], the formula is as follows:

$$
c^{t}=\frac{c^{t}}{I}, d^{t}=\frac{d^{t}}{I}, I=10^{w} \frac{t}{T}, w=\left\{\begin{array}{cc}
2 & t>0.1 T \\
3 & t>0.5 T \\
4 & t>0.75 T \\
5 & t>0.9 T \\
6 & t>0.95 T
\end{array}\right.
$$

This can effectively improve the rate of convergence.

When an ant-lion eats an ant, then it updates position to improve chances of catching ant, the formula is as follows:

$$
\text { Antlion }_{j}=\text { Ant }_{i}^{t} \quad f\left(\text { Ant }_{i}^{t}\right)>f\left(\text { Antlion }_{j}\right)
$$

During the iteration, all ants will be affected by the elite ant-lion, and the location of ants will be determined by roulette and random walk in order to reduce the local extremum [18, 19]:

$$
A n t_{i}^{t}=\frac{R_{A}^{t}+R_{E}^{t}}{2}
$$

Where $R_{A}^{t}$ is the choice of random walk of the roulette wheel in the $t$-th iteration $R_{E}^{t}$ is random walk of the ant around the elite ant lion in the $t$-th iteration.

\subsection{Extreme learning machine}

The input weights and hidden layer node offset of the extreme learning machine are selected randomly, and the hidden layer output weights are determined by the algorithm to minimize the training error [11-14].

Let $N$ sample sets are $\left\{\left(x_{j}, t_{j}\right), j=1,2, \ldots, N\right\}$, where $x_{\mathrm{j}}=\left[x_{\mathrm{j} 1}, x_{\mathrm{j} 2}, \ldots x_{\mathrm{j} n}\right]^{\mathrm{T}} \in \mathrm{R}^{\mathrm{n}}, t_{\mathrm{j}}=\left[t_{\mathrm{j} 1}\right.$, $\left.t_{\mathrm{j} 2}, \ldots \mathrm{t}_{\mathrm{jn}}\right]^{\mathrm{T}} \in \mathrm{R}^{\mathrm{m}}$, then ELM with $L$ hidden nodes can be expressed as:

$$
f_{L}\left(x_{j}\right)=\sum_{i=1}^{L} \beta_{i} g_{i}\left(\omega_{j}+b_{i}\right) \quad j=1,2, \ldots N
$$

where $\omega_{i}$ is the input weight of the $i$ hidden layer node, $\beta_{i}$ is the connection weight between the hidden layer node and the output node, $b_{i}$ is the bias vector of the hidden layer node, and $L$ is the number of hidden layer nodes[11, 12].A Matrix represents the output of the network is:

$$
\begin{gathered}
H \beta=T \\
\mathrm{H}=\left[\begin{array}{cccc}
g\left(\omega_{1} x_{1}+b_{1}\right) & g\left(\omega_{2} x_{1}+b_{1}\right) & \ldots & g\left(\omega_{L} x_{1}+b_{1}\right) \\
g\left(\omega_{1} x_{2}+b_{1}\right) & g\left(\omega_{2} x_{1}+b_{1}\right) & \ldots & g\left(\omega_{L} x_{1}+b_{1}\right) \\
\ldots & \ldots & & \\
g\left(\omega_{1} x_{\mathrm{N}}+b_{1}\right) & g\left(\omega_{2} x_{1}+b_{1}\right) & \ldots & g\left(\omega_{L} x_{1}+b_{1}\right)
\end{array}\right] \\
\beta=\left[\begin{array}{c}
\beta_{1}^{\mathrm{T}} \\
\beta_{2}^{\mathrm{T}} \\
\vdots \\
\beta_{L}^{\mathrm{T}}
\end{array}\right] \quad T=\left[\begin{array}{c}
T_{1}^{T} \\
T_{2}^{T} \\
\vdots \\
T_{N}^{T}
\end{array}\right]
\end{gathered}
$$

Where $H$ is the hidden layer output matrix, $\beta$ is the output weight matrix, $T$ is the expected output Matrix. The minimum error of $\|H \beta-T\|$ is calculated first, then the least square algorithm is used to obtain the minimum output weight $\hat{\beta}=H^{-1} T$. 


\subsection{The flow of ALO-ELM Prediction Algorithm}

The disadvantage of the extreme learning machine is that the input weights and the hidden layer deviation matrix are random, which affects the stability of the prediction results [14]. Ant-lion Algorithm was utilized to optimize the parameters of the extreme learning machine, to avoid the influence by random selection of parameters. The optimization function of the ALO-ELM Algorithm is described as:

$$
\begin{cases} & \min F(\omega, b) \\ \text { s.t. } & \omega \in(-1,1), b \in(-1,1)\end{cases}
$$

The flow diagram of the ALO-ELM prediction model is as follows:

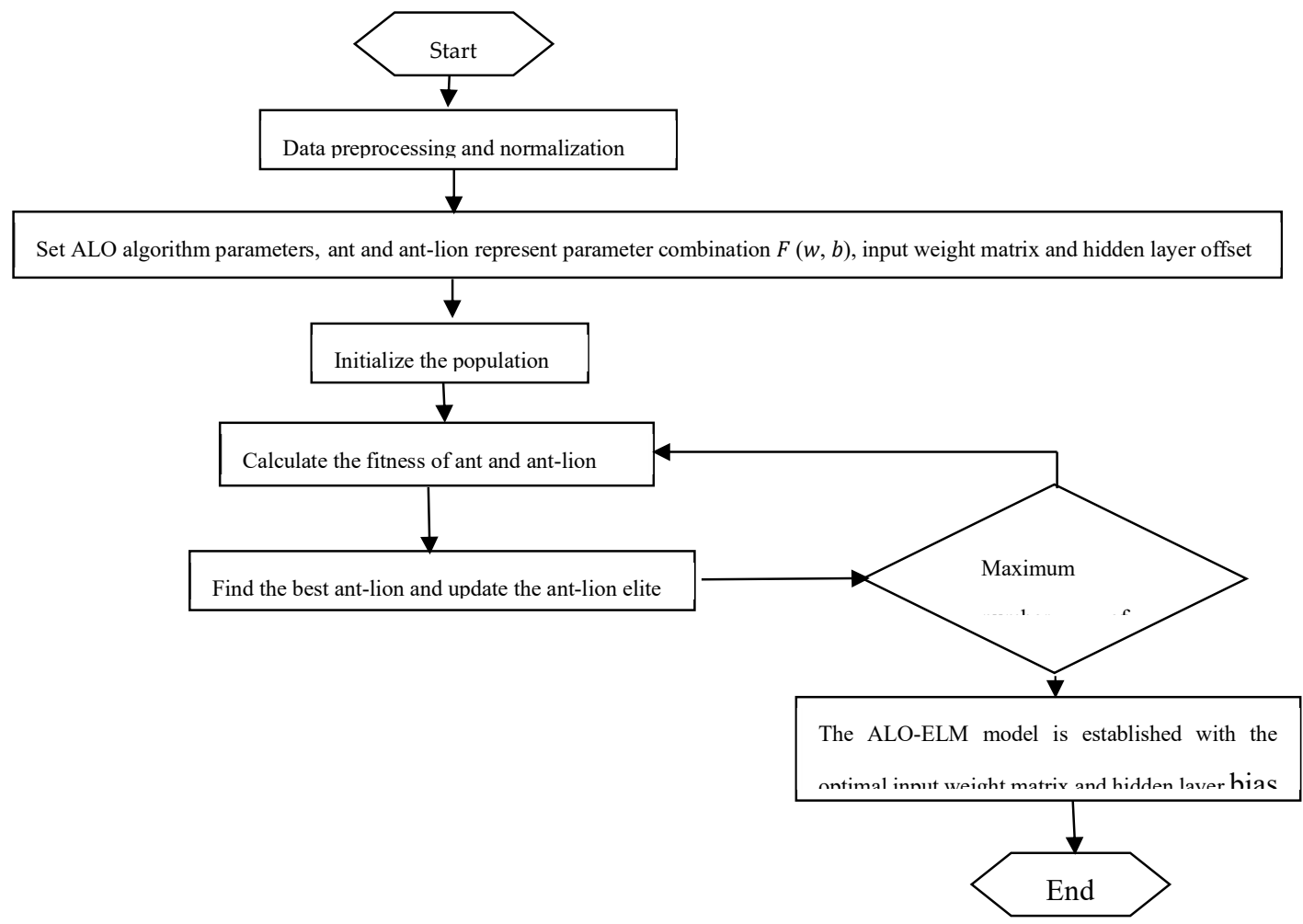

Figure 1. Flow chart of ALO-ELM algorithm

\section{Load Forecasting Based on Big Data}

The daily maximum load demand is not only related to historical data, but also influenced by temperature, day type (working day or holiday) and weather humidity. Based on the above factors and the historical daily maximum load demand of Chongqing in China from August 2019 to July 2020, a daily maximum load demand-forecasting model is established. The 10-day load demand data in August 2020 were selected as the test sets.

The parameters of ELM structure, input node parameters, hidden layer node parameters and output node parameters are optimized by ALO. Other parameters: maximum iteration times are 320, population sizes are 75, and excitation function is sigmoid type.

\section{Simulation Results and Discussion}

In order to verify the superiority of the ALO-ELM prediction model, the original ELM model was applied to forecast the maximum load demand of the same day, and then the comparison was made. Figure 2 is a comparison of the daily maximum load forecasting results of the two forecasting models in the validation data set. It can be seen that the predicted results of the two models are close to the actual load, but the ALO-ELM model has a better agreement with the actual load. Figure 3 shows the prediction error curve. As 
can be seen in Figure 3, the prediction error of ALO-ELM is less than that of ELM. Compared with the forecast results and forecast errors in Figure 2,Figute 3 and Table1, it is obvious that the daily maximum load demand predicted by the ALO-ELM model can match the actual demand very well, while the ELM forecast results have relatively large deviation, therefore, the ALO-ELM prediction model has higher prediction accuracy.

The forecast of daily maximum load demand is affected by many factors, and the variability of the forecast will lead to the forecast error between the forecast value and the actual value. The error evaluation index can evaluate the error level between the predicted value and the true value, and the average absolute error MAPE is selected as the evaluation index. MAPE is expressed as:

$$
M A P E=\frac{1}{N} \sum_{i=1}^{N}\left|\frac{F_{i}-R_{i}}{R_{i}}\right| \times 100 \%
$$

Where $N$ is the number of samples, $R_{\mathrm{i}}$ is the actual value, $F_{\mathrm{i}}$ is the predicted value.



Figure 2. Forecasting results of daily maximum load demand

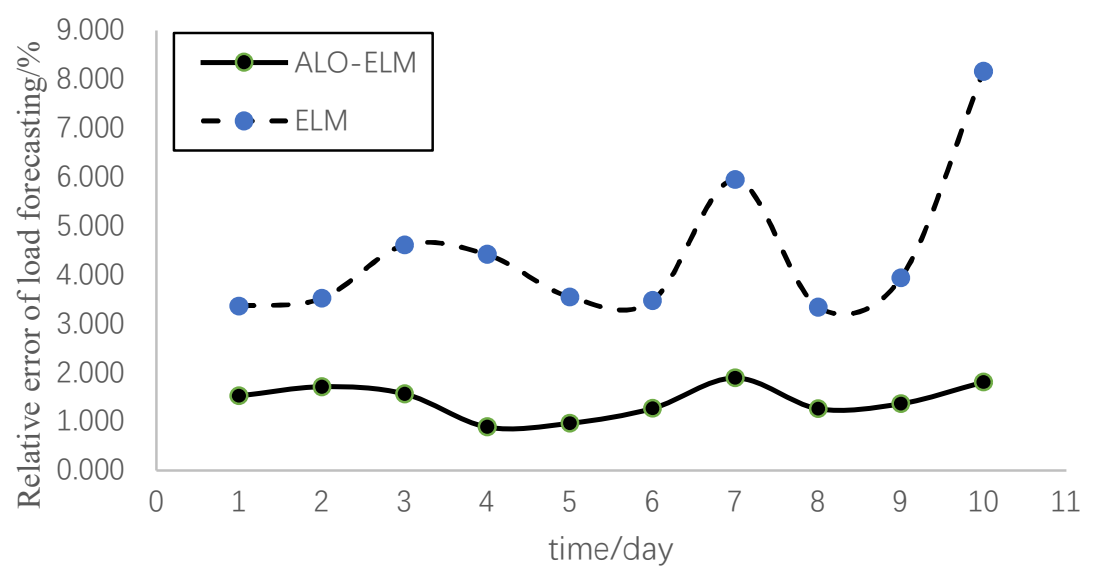

Figure 3. The load prediction error 
Table 1. Comparison of load forecasting by two methods

\begin{tabular}{cccccr}
\hline \multirow{2}{*}{ Data (2020) } & Actual load & \multicolumn{2}{c}{ ALO-ELM } & \multicolumn{2}{c}{ ELM } \\
& / MW & / MW & Relative error/\% & / MW & Relative error/\% \\
\hline August 1st & 2052.5 & 2021.2 & 1.525 & 1983.6 & 3.357 \\
August 2nd & 2047.4 & 2012.4 & 1.709 & 1975.5 & 3.512 \\
August 3rd & 2055.6 & 2087.7 & 1.562 & 2139.2 & 4.067 \\
August 4th & 1985.2 & 2002.8 & 0.887 & 2067.4 & 4.141 \\
August 5th & 1928.7 & 1947.3 & 0.964 & 1860.3 & 3.546 \\
August 6th & 1938.6 & 1914.1 & 1.264 & 2005.9 & 3.472 \\
August 7th & 2034.1 & 2072.6 & 1.893 & 2155.1 & 5.949 \\
August 8th & 2138.8 & 2165.8 & 1.262 & 2067.4 & 3.338 \\
August 9th & 1701.4 & 1724.6 & 1.364 & 1634.5 & 3.932 \\
August 10th & 1396.3 & 1421.5 & 1.805 & 1510.3 & 8.164 \\
\hline
\end{tabular}

Table 2. Prediction error analysis of two prediction models

\begin{tabular}{cc}
\hline Algorithm & MAPE / \% \\
\hline ALO-ELM & 1.41 \\
ELM & 4.34 \\
\hline
\end{tabular}

From the error analysis of Table 1 and Table 2, it can be seen that MAPE values of both forecasting models are small. The average absolute error of short-term load demand predicted by ALO-ELM model is 1.41, while that predicted by ELM is 4.34, obviously the mean absolute error of ALO-ELM model is lower. The prediction accuracy of the improved ELM is higher as shown in Table 2 and Figure 2, which shows the validity of the proposed ALO-ELM prediction method.

\section{Conclusion}

The Ant-lion Optimizer was applied to optimize the input weights and hidden-layer node offset of Extreme Learning Machine, and short-term load forecasting model based on ALO-ELM combined method was established in this paper. To train and verify the superiority and validity of the ALO-ELM forecast model, the practical daily maximum load demand of Chongqing city in one-year are illustrated it. Compared with those predicted by the basic extra learning machine model, the results show that:

1) The daily maximum load demand predicted by the ALO-ELM model is in good agreement with the actual demand, while the ELM prediction results have a relatively deviation;

2) Both models have small MAPE values. The mean absolute error of short-term load demand predicted by ALO-ELM is 1.41, while that predicted by ELM is 4.34, obviously, the ALO-ELM model has a lower mean absolute error.

Author Contributions: software, Wan Zou; formal analysis, Xiangxi Duan ; writing - original draft preparation, $\mathrm{Yi} \mathrm{Li} \mathrm{;} \mathrm{writing} \mathrm{-} \mathrm{review} \mathrm{and} \mathrm{editing,} \mathrm{Ming} \mathrm{He.} \mathrm{All} \mathrm{authors} \mathrm{have} \mathrm{read} \mathrm{and} \mathrm{agreed} \mathrm{to} \mathrm{the}$ published version of the manuscript.

Funding: This research was funded by State Grid science and technology projects (No. SGTYHT/19-JS215).

\section{References}

1. Almeshaiei E, Soltan H. A methodology for electric power load forecasting. Alexandria Engineering Journal. 2011, 50(2), 137-144.

2. Liu Z., Sun W., Zeng J. A new short-term load forecasting method of power system based on EEMD and SS-PSO [J]. Neural Computing and Applications, 2014, 24(3-4), 973-983. 
3. Mukherjee, D., Chakraborty, S., Ghosh, S., and Mishra, R. K.. Application of deep learning for power system state forecasting. International Transactions on Electrical Energy Systems.2021, 5, 1-26.

4. Baesmat, K. H., Masodipour, I., and Samet, H.. Improving the performance of short-term load forecast using a hybrid artificial neural network and artificial bee colony algorithm. Canadian Journal of Electrical and Computer Engineering, 2021, 44(3), $275-282$.

5. P. Duan, K. Xie, T. Guo, X. Huang. Short-term load forecasting for electric systems using the PSO-SVR and FCM Clustering Techniques. Energies, 2011, 4, 173-184.

6. Khoa, T., Phuong, L. M., Binh, P., and Lien, N.. Application of wavelet and neural network to long-term load forecasting. International Conference on Power System Technology, IEEE Xplore 2004, 840-844.

7. X.Sun and X.Zhang.Short term load forecasting using core vector regression trained with particle swarm optimization. 2016 international conference on computer engineering and information systems, Advances in Computer Science Research, volume 52, 300-304.

8. W.Zhang, W.Ma, G.Yong and Z.Dong. Short-term power load forecasting based on optimized KELM. Chinese Journal of Computer Simulation, 2019, 36(10), 125-128+135.

9. Y.Bi, Yunfan,A.Han, Z.Zhang and W.Sun.Study on short-term load forecasting model based on fuzzy bagging-GBDT. Chinese Journal of Proceedings of the CSU-EPSA, 2019, 31(07), 51-56.

10. Y.He, Q.Xu. Short-term power load forecasting based on self-adapting PSO-BP neural network model. Fourth International Conference on Computational and Information Sciences, 2012, 1096-1099.

11. Huang G. B., Zhu Q. Y. S. Extreme learning machine: a new learning scheme of feedforward neural networks. International Joint Conference on IEEE, 17 January 2005, 985-990.

12. Li Y. B., Liu F. and Cao Q. A review of the research on the prediction model of extreme learning machine. Conference Series of 2019 2nd International Conference on Advanced Algorithms and Control Engineering, Guilin, 2019, Journal of Physics,1-9.

13. Li Y. B., Liu F. Combined kernel KELM prediction model based on quantum particle swarm optimization. Proceedings of 2019 IEEE 3rd Information Conference on Electronic Information Technology and Computer Engineering, Xiamen, 2019, 136-139.

14. Lv F.,Liu Y.,Qi Y.,Yan Y.,Zhang J. and Xie Q..Short-term load forecasting based on optimized learning machine using improved genetic algorithm. Journal of North China Electric Power University, 2018, 45(6), 1-7.

15. Cao J., Lin Z. Self-adaptive evolutionary extreme learning machine. Neural Processing Letters, 2012, 36(3), $285-305$.

16. Li W., ChenY., Guo K.,Guo S,Liu Z.. Parallel extreme learning machine based on improved particle swarm optimization. Chinese Journal of Pattern recognition and artificial intelligence (PREAI), 2016, 29(9), 840-849.

17. Mirjalili S.The Ant lion optimizer. Advances in Engineering Software, 2015, 83(C), 80-98.

18. E.S. Ali, S.M. Abd Elazim, A.Y. Abdelaziz. Ant Lion Optimization Algorithm for Renewable Distributed Generations. Energy, 2016(116), 445-458.

19. K. Roy,K.K. Mandal,A.C. Mandal. Ant-Lion Optimizer algorithm and recurrent neural network for energy management of micro grid connected system. Energy, 167 (2019), 402-416. 